\title{
Leads leading leaflets to leak
}

Today, approximately 1 million new permanent pacemakers (PPMs) are implanted in patients annually worldwide. ${ }^{1}$ Increasing cases of transcatheter aortic valve replacement and the use of rapid deployment valves might augment the number of PPM implantations. Among the broad spectrum of complications involving endocardial PPM implantation, tricuspid regurgitation (TR), which requires surgical intervention, is no longer a rarely encountered complication, because the tricuspid valve (TV) is no longer neglected as it had been before. However, there is a paucity of literature on the subject.

In 1980, Gibson and colleagues ${ }^{2}$ reported the first PPM-induced TV malfunction, and in 2013, Al-Bawardy and colleagues ${ }^{3}$ published a comprehensive review on TR in patients with PPMs and implantable cardioverterdefibrillators. In that review, the authors summarized that device-related TR was usually due to mechanical (perforation/laceration of leaflets, entrapment of leads resulting in scar tissue, or interference with valve coaptation) or physiologic (asynchronized activation of the right ventricle from apex to base) mechanisms.

In this issue of the Journal, Saran and coworkers ${ }^{4}$ have analyzed the results of TV surgeries in the presence of PPMs collected during the past 2 decades at their institution. In this retrospective study, the authors have defined 2 concepts: pacemaker-associated TR (PATR) and pacemaker-induced TR (PITR). In PATR, TR is not directly caused by PPM leads, whereas in PITR it is. According to their definitions, 622 patients were reviewed and 598 were enrolled, 349 in the PATR group and 249 in the PITR group. The most common cause of PATR was functional TR (as expected), and TV repair rate was approximately $62 \%$. The authors classified the mechanisms of PITR into 5 categories: (1) restricted mobility of leaflets, (2) adherent leaflet to lead, (3) leaflet perforation, (4) scarring of leaflets, and (5) chordal entrapment. In general, with the exception of extensively destructed account. techniques. is promising. ${ }^{1}$

\section{References}

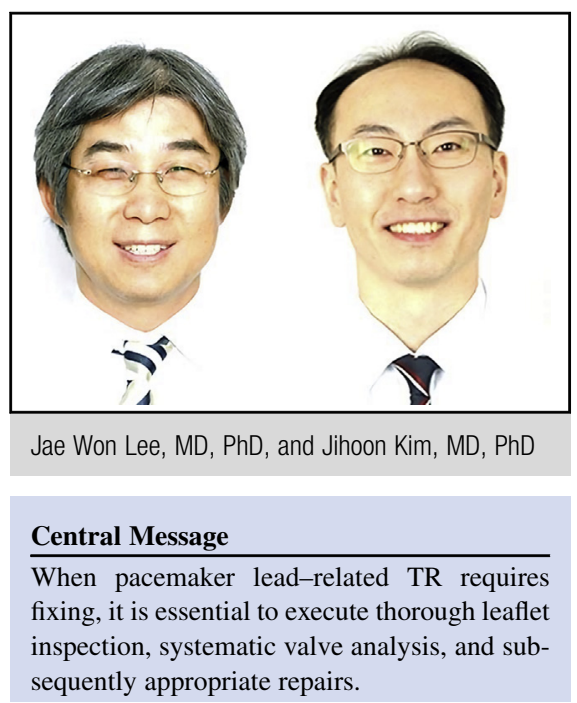

See Article page 1498.

valve leaflets, the majority of TV lesions are repairable. The fundamental principle of TR repair in PITR (especially in "restricted mobility of leaflets") is putting aside the lead from the valve leaflets and subvalvular apparatus so that valve motions can be free of impediments. In addition, the lead should not be fastened completely, considering the possibility of its future removal. At the time of the operation, when removing the endocardial lead, epicardial lead implantation also should be taken into

As Saran and coworkers ${ }^{4}$ have stated, TV replacement was an independent risk factor for mortality; therefore, we have to do our best to repair the valve. TV leaflets often can be difficult to inspect because of their anterior to posterior facing anatomy; yet, the key to success is thorough inspection of the valve leaflets and the subvalvular apparatus. In the absence of a standardized technique to repair PATR/PITR, further studies are warranted to evaluate the outcomes of corresponding repair

Currently, left ventricular pacing by lead insertion via coronary sinus is considered feasible and safe after TV surgery. ${ }^{5}$ The recent introduction of "leadless" pacemakers, which are virtually free from lead-related complications,

1. Tjong FV, Reddy VY. Permanent leadless cardiac pacemaker therapy: a comprehensive review. Circulation. 2017;135:1458-70. 
2. Gibson TC, Davidson RC, Desilvey DL. Presumptive tricuspid valve malfunction induced by a pacemaker lead: a case report and review of the literature. Pacing Clin Electrophysiol. 1980;3:88-94.

3. Al-Bawardy R, Krishnaswamy A, Bhargava M, Dunn J, Wazni O, Tuzcu EM, et al. Tricuspid regurgitation in patients with pacemakers and implantable cardiac defibrillators: a comprehensive review. Clin Cardiol. 2013;36:249-54
4. Saran N, Said SM, Schaff HV, Maltais S, Stulak JM, Greason KL, et al. Outcome of tricuspid valve surgery in the presence of permanent pacemaker. J Thorac Cardiovasc Surg. 2018;155:1498-508.

5. Sideris S, Drakopoulou M, Oikonomopoulos G, Gatzoulis K, Stavropoulos G, Limperiadis D, et al. Left ventricular pacing through coronary sinus is feasible and safe for patients with prior tricuspid valve intervention. Pacing Clin Electrophysiol. 2016;39:378-81. 